\title{
Constraints on Temporal Variations in Velocity near Anza, California, from Analysis of Similar Event Pairs
}

\author{
by Jennifer S. Haase, Peter M. Shearer, and Rick C. Aster
}

\begin{abstract}
Similar earthquake pairs recorded by the Anza Seismic Network in southern California are used as repeatable sources to place an upper limit on temporal changes in seismic velocity which occurred in the vicinity of the Anza seismic gap in the last $9 \mathrm{yr}$. Relative arrival times for each pair of events are found using a cross-correlation method and relative locations are calculated to verify that the pairs have nearly identical hypocenters. The time separation between events in these pairs varies from less than a day to almost 7 yr. The longterm changes in seismic travel times, as measured from the pairs with the longest time separation, are not significantly greater than the noise level estimated from the short-time-separation event pairs. Almost all $P$-wave paths show less than $0.06 \%(0.007 \mathrm{sec})$ change in travel time and all $S$-wave paths have less than $0.03 \%(0.004 \mathrm{sec})$ change. Sensitivity tests place an upper bound on traveltime changes that could be compensated by hypocenter mislocation at $0.2 \%$. There is no evidence that localized stress accumulation causes measurable changes in seismic velocity in the Anza region.
\end{abstract}

\section{Introduction}

If temporal changes in seismic velocity within the crust could be observed, they would provide valuable clues regarding stress variations and earthquake processes. This is because velocity is sensitive to changes in the crack properties of fractures present in the rock which could accompany stress changes at depth. These might be widespread long-term changes resulting from the gradual accumulation of stress, but the greatest interest has been in the possibility of localized precursory velocity changes immediately before earthquakes because of their potential as an earthquake prediction tool. In the 1970s great excitement accompanied the apparent discovery of large (10 to $20 \%$ ) velocity changes before several earthquakes (e.g., Semenov, 1969; Aggarwal $e t$ al., 1973, 1975; Whitcomb et al., 1973; Robinson et al., 1974). These observations were a key part of the shortlived dilatancy theory of earthquake prediction (e.g., Nur, 1972; Scholz et al., 1973; Anderson and Whitcomb, 1975), and were based on analyses of arrival times from natural seismic events. However, it soon became apparent that artificial sources with repeatable locations and waveforms offered a far more accurate method of monitoring possible velocity changes. Studies of records from quarry blasts and nuclear explosions found no evidence for velocity changes before earthquakes down to levels of 1 to $2 \%$ (e.g., McEvilly and Johnșon, 1974; Boore et al., 1975; Kanamori and Fuis, 1976; Bolt, 1977; Chou and Crosson, 1978). Repeated measurements of travel times from an air gun deployed near the San Andreas fault showed no changes greater than $0.1 \%$ over a $2-y r$ period (Leary et al., 1979; Leary and Malin, 1982).

These limits are an order of magnitude smaller than the changes that were claimed for the natural earthquake studies. The likely cause for this discrepancy is that the nonrepeatability of earthquake sources makes it difficult to measure accurately any changes in velocity along the source-receiver ray paths. Lindh et al. (1978) showed that apparent premonitory changes in velocity before two earthquakes in California could be explained by differences in earthquake depth and magnitude unrelated to any change in velocity preceding the events. The difficulties associated with the nonstationarity of natural seismic sources can be greatly reduced if similar earthquake pairs can be identified that have nearly identical locations and waveforms (Poupinet et al., 1984). For these events, cross correlation of similar waveforms allows extremely accurate relative timing of arrivals and event relocation. Studies of similar earthquake pairs spanning the time of the 1979 Coyote Lake earthquake show an apparent time shift in the $S$-wave coda, consistent with a $0.2 \%$ decrease in the shear velocity at the time of the event in a 5- to 10-km-wide region (Poupinet et al., 1984; Ellsworth et al., 1987). A similar study of event pairs spanning the 1979 Loma Prieta earthquake finds evidence for an apparent time shift in the $S$-wave coda, consistent with a $0.3 \%$ decrease in shear velocity within the 
aftershock region (Ellsworth et al., 1992; Ellsworth, personal comm.).

Velocity differences following major earthquakes are not unexpected because of the large changes in static stresses near the fault. Of greater interest for earthquake prediction would be the occurrence of changes prior to the events. The Ellsworth et al. (1992) study found no changes greater than $0.05 \%$ in the direct $S$ velocity in a 10-yr period leading up to the Loma Prieta earthquake. However, they found some evidence for time shifts in the $S$-wave coda which might be caused by a slight decrease in the average $S$ velocity before the earthquake. A study using similar microearthquakes in the Parkfield nucleation zone to detect changes in travel times and polarizations of $P$ - and $S$-wave arrivals over the $4+$ yr of operation of the high-resolution network did not detect a significant change within the resolution of the technique (Nadeau et al., 1992). Additional studies of this type are needed to determine whether temporal velocity changes can be seen reliably and if they are widespread, and to distinguish between long-term changes resulting from the gradual accumulation of stress and any anomalous changes that might precede major earthquakes.

It should be noted that the presence of precursory phenomena is not required by many models of earthquake rupture. The decrease in the observed sizes of

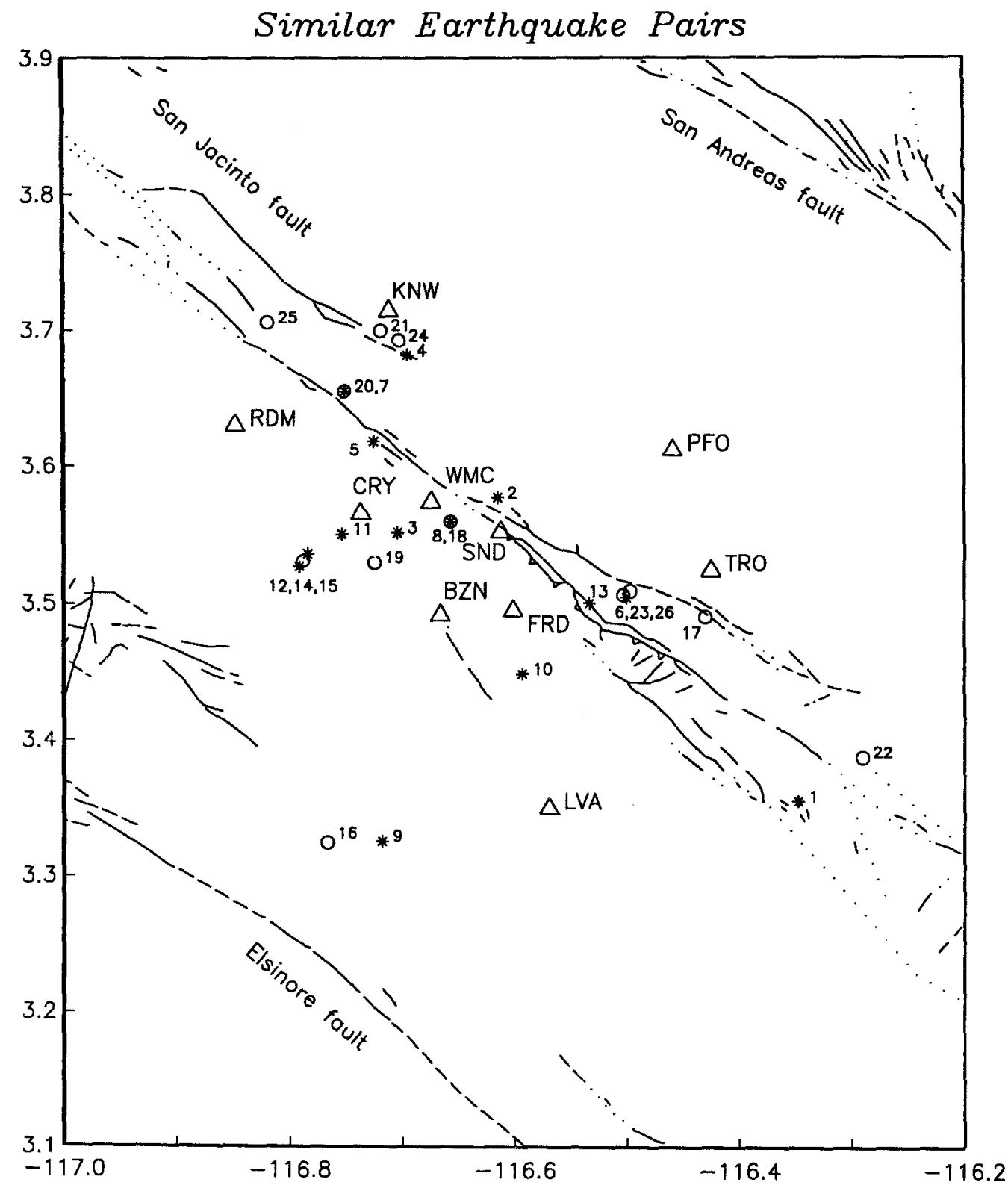

Figure 1. Selected similar earthquake pairs that occurred in the vicinity of the Anza Seismic Gap in the San Jacinto fault zone in southern California. $\mathrm{O}=$ event pair with time separation $\delta T>290$ days, $*=$ event pair with $\delta T<50$ days, $\Delta=$ Anza Seismic Network station. Reference numbers refer to Table 1. 
possible precursory velocity changes has been accompanied by an increase in discussion of earthquake models that are inherently unpredictable (Brune, 1979; Kagan and Knopoff, 1981, 1987; Rundle, 1988; Bak and Tang, 1989). In these models of a complex system near failure, large earthquakes are triggered by smaller earthquakes, and, in the limit, the region in which precursory signals might be expected is vanishingly small. Even if every small event could be predicted, the problem becomes predicting which of the many small earthquakes will grow into a large earthquake. In a rupture zone of existing heterogeneous stress, a small perturbation to the stress field not necessarily near the zone of the greatest moment release may determine whether rupture initiates or continues, leading to the possibility that earthquakes may not have any premonitory signals at all.

In this article, we analyze similar earthquake pairs recorded over a 9-yr period by the Anza Seismic Network along the San Jacinto fault in southern California. This region is well suited for this experiment because of the ready availability of three-component digital waveforms from thousands of small events, the proximity to strain meters at Pinyon Flat Observatory, and the relatively high probability of a future magnitude 7 earth- quake near Anza (Working Group on California Earthquake Probabilities, 1988). Crampin et al. (1990) reported large temporal changes in shear-wave splitting delay times from one station of the Anza network associated with the $1986 M_{L}=5.6$ North Palm Springs event about 30 $\mathrm{km}$ north of the network. However, a detailed study of shear-wave splitting delay times at Anza using an automated procedure failed to reproduce the Crampin et al. results (Aster et al., 1990), and analysis of similar event pairs showed that any changes in delay times must be at least an order of magnitude smaller than those claimed by Crampin et al. (Aster et al., 1990, 1991). We do not directly reexamine this issue here, but focus on constraining the size of any temporal change in the initial $P$ - and $S$-wave arrivals in the Anza region and assessing the reliability of these constraints given the limited data available. We will also address the possibility of co-seismic changes in average velocity in the Anza region associated with large earthquakes at regional distances.

\section{Data}

The Anza Network data consist of high-dynamicrange $(96 \mathrm{~dB})$ high-frequency $(2$ to $62.5 \mathrm{~Hz})$ three-com-

Table 1

Selected Similar Earthquake Pairs That Occurred in the Vicinity of the Anza Seismic Gap in the San Jacinto Fault Zone in Southern California*

\begin{tabular}{|c|c|c|c|c|c|c|c|c|c|}
\hline $\begin{array}{c}\text { Pair } \\
\text { Number }\end{array}$ & $\begin{array}{c}\text { Event } 1 \\
\text { Date }\end{array}$ & $M_{L}$ & $\begin{array}{c}\text { Event } 2 \\
\text { Date }\end{array}$ & $M_{L}$ & Longitude & Latitude & $\begin{array}{l}\text { Depth } \\
(\mathrm{km})\end{array}$ & $\begin{array}{l}\delta X \\
(\mathbf{m})\end{array}$ & $\begin{array}{c}\delta T \\
\text { (days) }\end{array}$ \\
\hline 1 & 84128141918 & 2.5 & 84128141536 & 2.3 & -116.34723 & 33.35440 & 11.72 & 86 & 0 \\
\hline 2 & 91094173152 & - & 91094182746 & 一 & -116.61450 & 33.57703 & 12.48 & 44 & 0 \\
\hline 3 & 82331042239 & 2.0 & 82331121058 & 2.2 & -116.70383 & 33.55113 & 6.58 & 58 & 0 \\
\hline 4 & 86290230031 & 1.6 & 86290232427 & 1.5 & -116.69453 & 33.68151 & 16.67 & 31 & 0 \\
\hline 5 & 91301100941 & - & 91301101834 & - & -116.72486 & 33.61825 & 14.85 & 49 & 0 \\
\hline 6 & 90263094332 & 1.5 & 90263094414 & 1.6 & -116.50033 & 33.50419 & 14.13 & 50 & 0 \\
\hline 7 & 88279220545 & - & 88279220644 & 1.9 & -116.75035 & 33.65524 & 14.80 & 12 & 0 \\
\hline 8 & 84177184349 & - & 84177184411 & 1.4 & -116.65680 & 33.55912 & 6.87 & 25 & 0 \\
\hline 9 & 83285103701 & 2.4 & 83285103746 & 1.9 & -116.71812 & 33.32539 & 12.55 & 51 & 0 \\
\hline 10 & 84207125717 & 1.4 & 84207125939 & 1.6 & -116.59317 & 33.44881 & 10.18 & 24 & 0 \\
\hline 11 & 88118222210 & 1.7 & 88121000309 & 1.5 & -116.75317 & 33.55021 & 2.60 & 74 & 3 \\
\hline 12 & 84191151716 & 1.5 & 84207221148 & 1.4 & -116.79113 & 33.52670 & 0.77 & 13 & 16 \\
\hline 13 & 84158040344 & 1.6 & 84191100614 & 2.7 & -116.53324 & 33.50014 & 10.10 & 76 & 33 \\
\hline 14 & 85011151207 & 1.9 & 85057024642 & 1.8 & -116.78385 & 33.53564 & 3.78 & 37 & 46 \\
\hline 15 & 84207221148 & 1.4 & 85134220533 & 1.6 & -116.78788 & 33.53059 & 0.65 & 49 & 292 \\
\hline 16 & 86240150620 & 2.2 & 87197222209 & 1.9 & -116.76661 & 33.32443 & 7.93 & 132 & 322 \\
\hline 17 & 86128022635 & 2.1 & 87200002952 & 1.8 & -116.42989 & 33.49013 & 7.58 & 22 & 437 \\
\hline 18 & 84177184349 & - & 85266064103 & 2.1 & -116.65685 & 33.55927 & 6.90 & 53 & 454 \\
\hline 19 & 84306203243 & 0.8 & 86287175236 & 1.8 & -116.72455 & 33.52949 & 1.64 & 17 & 711 \\
\hline 19 & 84306203243 & 1.4 & 86287175236 & 1.8 & -116.72455 & 33.52949 & 1.64 & 17 & 711 \\
\hline 20 & 88279220545 & - & 90263174616 & 1.6 & -116.75068 & 33.65511 & 14.83 & 34 & 714 \\
\hline 21 & 84203235348 & 1.9 & 86218054938 & 2.9 & -116.71810 & 33.69973 & 17.88 & 58 & 745 \\
\hline 22 & 84175093325 & 2.5 & 87024033152 & 2.4 & -116.28984 & 33.38673 & 12.03 & 39 & 944 \\
\hline 23 & 86287100614 & 2.5 & 90105071049 & 1.2 & -116.49669 & 33.50859 & 14.68 & 42 & 1279 \\
\hline 24 & 87307221107 & 1.6 & 91274052047 & 1.5 & -116.70187 & 33.69246 & 18.12 & 34 & 1428 \\
\hline 25 & 82320203840 & 2.1 & 88130220747 & - & -116.81843 & 33.70576 & 15.54 & 32 & 2001 \\
\hline 26 & 84139101553 & 1.7 & 91094095001 & 1.6 & -116.50280 & 33.50573 & 13.94 & 11 & 2512 \\
\hline
\end{tabular}

* Reference numbers correspond to events plotted in Figure 1. Longitude and latitude are given for the first event of the pair. $\delta X$ is the distance between the events and $\delta T$ is the time difference between the events ( 0 means less than 1 day). 
ponent velocity records recorded at 250 samples per second (Vernon, 1989; Berger et al., 1984). Our search for similar event pairs began with the automatically calculated similar earthquake catalog for the Anza Network of Aster and Scott (1993), covering the period from 1 October 1982 through 14 April 1992. From this comprehensive catalog, we selected event pairs in which two or more stations of the network recorded waveforms with three-component cross-correlation coefficients greater than 0.9 for a 0.512 -sec window containing the $P$-wave ar- rival. The degree of waveform similarity varies greatly between these event pairs, and the vast majority of such pairs are of very short time separation and are thus of limited use in measuring possible long-term changes. However, we were able to identify a relatively small number of high-quality pairs with significant time separation.

We examined 12 pairs of very similar earthquakes occurring in the vicinity of the Anza seismic gap, which were separated in time by more than 290 days, with the
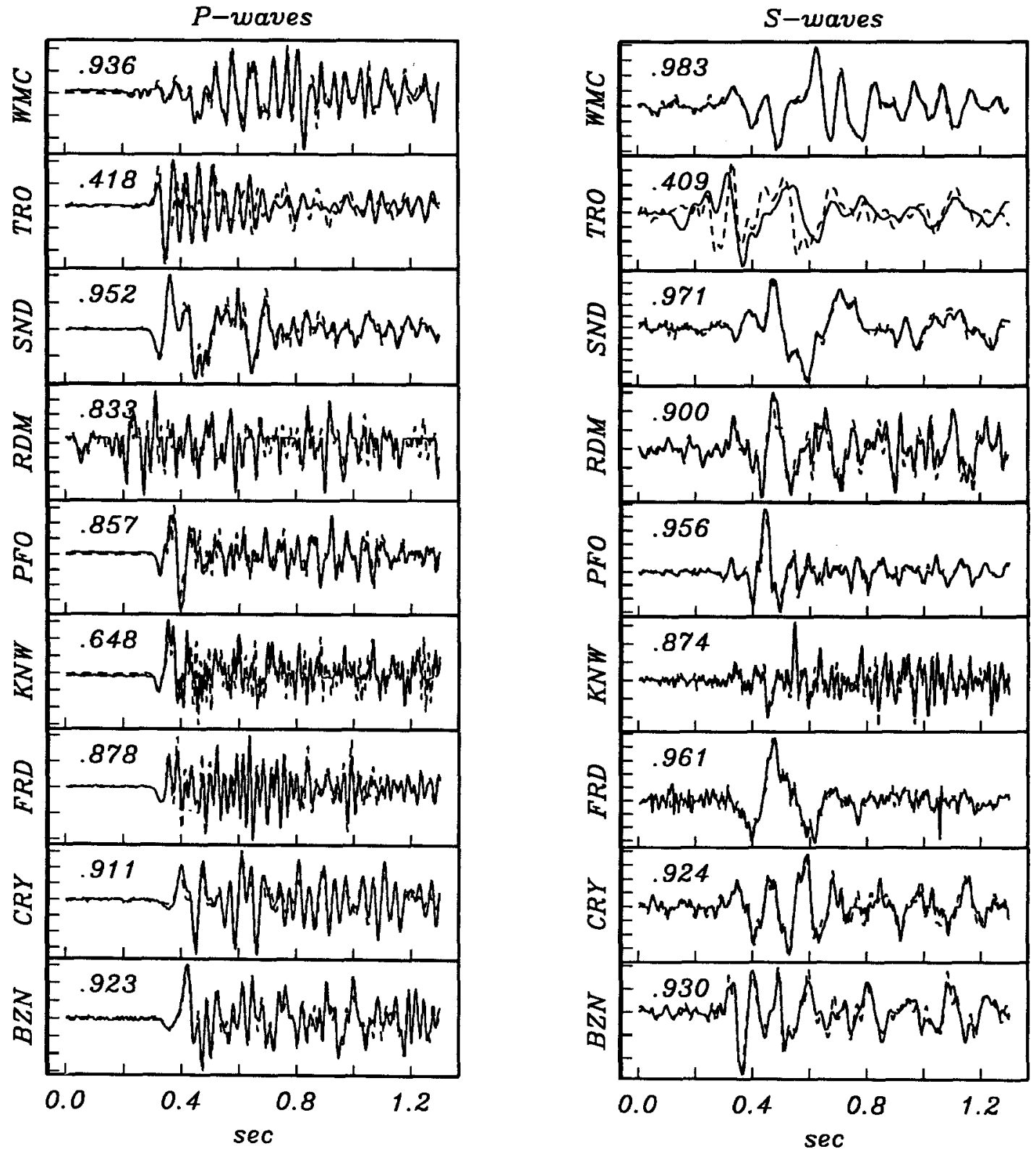

Figure 2. Nearly identical unfiltered $P$ and $S$ waveforms for event pair number 26 (time separation of 2512 days). Event 84139101553 is shown with solid lines, event 91094095001 is shown with dashed lines. The vertical component is plotted for the $P$ waves, while one of the two horizontal components is plotted for the $S$ waves. The peak cross-correlation value is given in the upper left corner. Station TRO was moved $\sim 50 \mathrm{~m}$ between the two events so shows lower correlation coefficients. 
greatest time separation being 2512 days. We also found 14 pairs of similar earthquakes with a time separation of less than 46 days (most with time separations of less than a day), which sample approximately the same ray paths as the pairs with larger time separations. As the time separation of these pairs is short enough that a significant velocity change due to accumulating stress between the events is unlikely, these event pairs can serve as a baseline measurement of the source repeatability and correlation noise. The locations of the earthquake pairs are shown in Figure 1 and are listed in Table 1. The wave- forms for the event pair with a 2512-day separation, plotted in Figure 2, demonstrate the high degree of waveform similarity present in the dataset. Figure 3 shows the waveforms for an event pair with approximately the same source location as the pair in Figure 2, but separated by about $45 \mathrm{sec}$.

After culling these events from the catalog, the relative arrival times of the $P$ - and $S$-wave phases were picked using time domain cross correlation in a procedure which allowed us to choose the initial phase alignment and fit window interactively. For each component
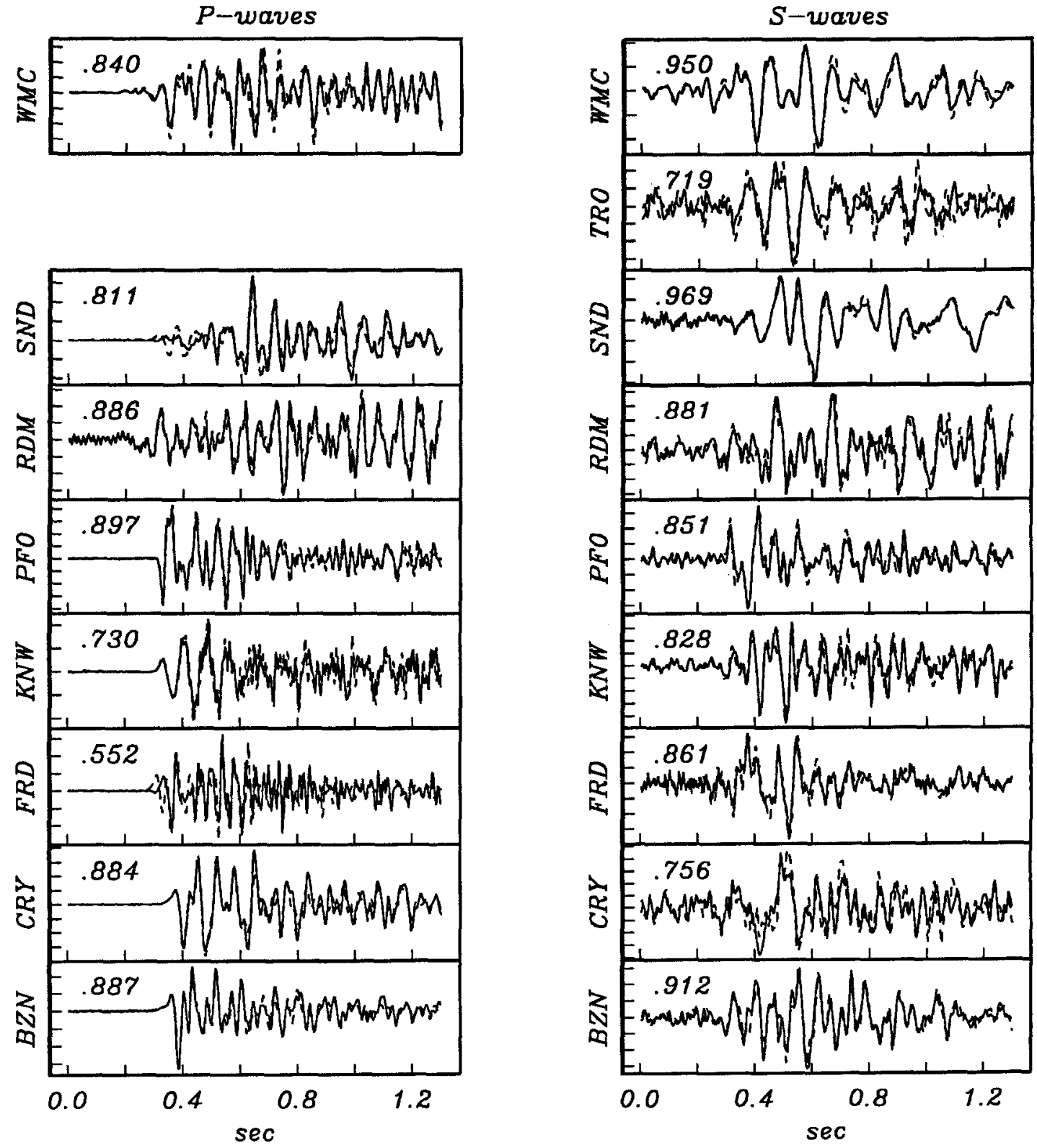

Figure 3. Nearly identical waveforms for event pair number 6 (time separation of about $45 \mathrm{sec}$ ). This pair is very close to event pair number 26 shown in Figure 2. Event 90263094332 is shown with solid lines, event 90263094414 is shown with dashed lines. The $P$ wave was not recorded at station TRO. The peak cross-correlation value is given in the upper left corner. 
of motion, the cross-correlation function maximum was found to an accuracy of 1 sample and then the traces were time shifted in the frequency domain to obtain a timing resolution of a tenth of a sample $(0.0004 \mathrm{sec})$. Because incoherency due to small-scale scattering might be a greater problem at higher frequencies, we experimented with low-pass filtering the seismograms to see what effect this would have on our results. In general, the low-pass records could be fit to higher correlation coefficients but with a corresponding loss in time resolution. This is illustrated in Figure 4, which plots the correlation coefficient as a function of time offset for the $P$ wave for an event pair recorded at station $\mathrm{KNW}$ with unfiltered data and data low-pass filtered at $24 \mathrm{~Hz}$. The peak for the filtered trace is higher and broader than the peak for the original data, and there is a slight time shift between the peaks. We chose to use the unfiltered data in our analysis because the times appeared to be more accurate, but the results discussed below would be very similar for the filtered data since the time shift between the two methods is generally less than $0.003 \mathrm{sec}$ and the unfiltered picks are within the larger error estimates of the filtered picks.

The correlation coefficient obtained for each pair of waveforms varies somewhat with the size of the fit win-

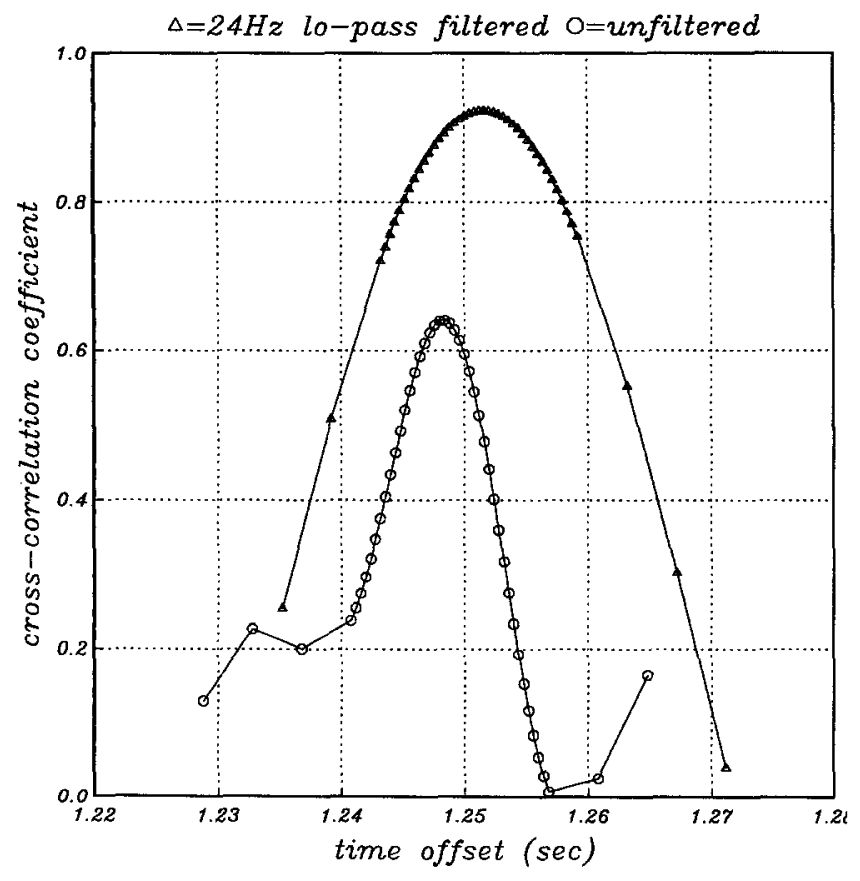

Figure 4. Cross-correlation function for event pair number 26 recorded at station $\mathrm{KNW}$. $\mathrm{O}=$ unfiltered data, $\Delta=$ data low-pass filtered at 24 $\mathrm{Hz}$. Widely spaced points indicate cross-correlation function with 1-sample accuracy. Closely spaced points indicate refined estimate of crosscorrelation function with 0.1 sample accuracy. The filtered data have a higher peak cross-correlation value, but the unfiltered data have a narrower peak and thus produce a more accurate lag estimate. dow selected for each station. The length of the fit window was 0.8 to $1.1 \mathrm{sec}$. The median correlation coefficient for the 26 event pairs is 0.88 for $P$ waves and 0.95 for $S$ waves. The correlation is significantly higher for the $S$ waves because of their higher signal-to-noise ratio and their lower frequency content, i.e., less incoherent high-frequency scattered energy. For most of the data, we estimate that the accuracy of the picks is about $0.0016 \mathrm{sec}$, using the width at which the correlation function is $90 \%$ of the height of the peak as a measure of the method precision. Because the data were not sampled synchronously at all stations before 1989 (Frank Vernon, personal comm.), there is an additional probable timing uncertainty of $\pm 1 / 2$ sample. This issue will be investigated more fully when we discuss constraining temporal variations in $S-P$ times.

\section{Results}

Two events with exactly the same hypocenter should show no variation in relative arrival times across the array if the velocity in the portion of the sampled medium has remained constant. The relative arrival times in this

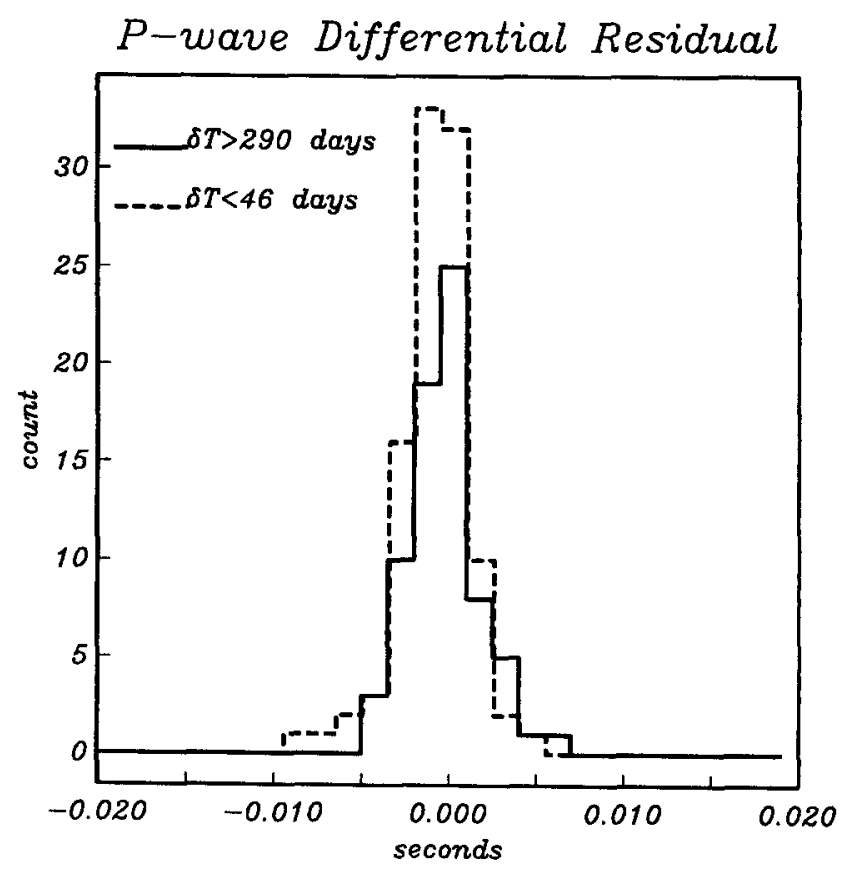

(a)

Figure 5. (a) Histograms of differential $P$-wave travel-time residuals for the subset of event pairs with $\delta T>290$ days (solid line) and the subset of event pairs with $\delta T<46$ days (dashed line). These residuals are corrected for offsets in event origin times and for differences in event locations. The standard deviations are $\sigma=0.002 \mathrm{sec}$ for both $P$-wave residual distributions. 
case should simply be the difference in origin times. We take into account the possibility of slight differences in hypocenter locations by calculating the relative distance between the two events using a joint hypocenter determination routine similar to that of Fremont and Malone (1987), Jordan and Sverdrup (1981), and Ito (1985), which minimizes the $L_{2}$ norm of the differential travel-time residuals in a linearized inversion. The variations in differential travel times due to slight location differences can then be removed, leaving differential travel-time residuals that can be explained either by a change in velocity or by random noise in the measurement procedure. The computed interhypocenter separations for our event pairs are listed in Table 1. These separations are less than $90 \mathrm{~m}$, except for one pair with hypocenter separation of $132 \mathrm{~m}$, which was included because of the exceptionally high coherence of the waveforms. The accuracy of the relative locations is less than $10 \mathrm{~m}$; the events resolvably did not occur in exactly the same places.

Our assumption is that the travel-time residuals for the pairs of events with very short time separations are due to random errors, and that only those residuals from the larger time separation pairs that exceed this error level may be reasonably attributed to a cumulative change in the seismic velocity of the medium. Figure 5a compares

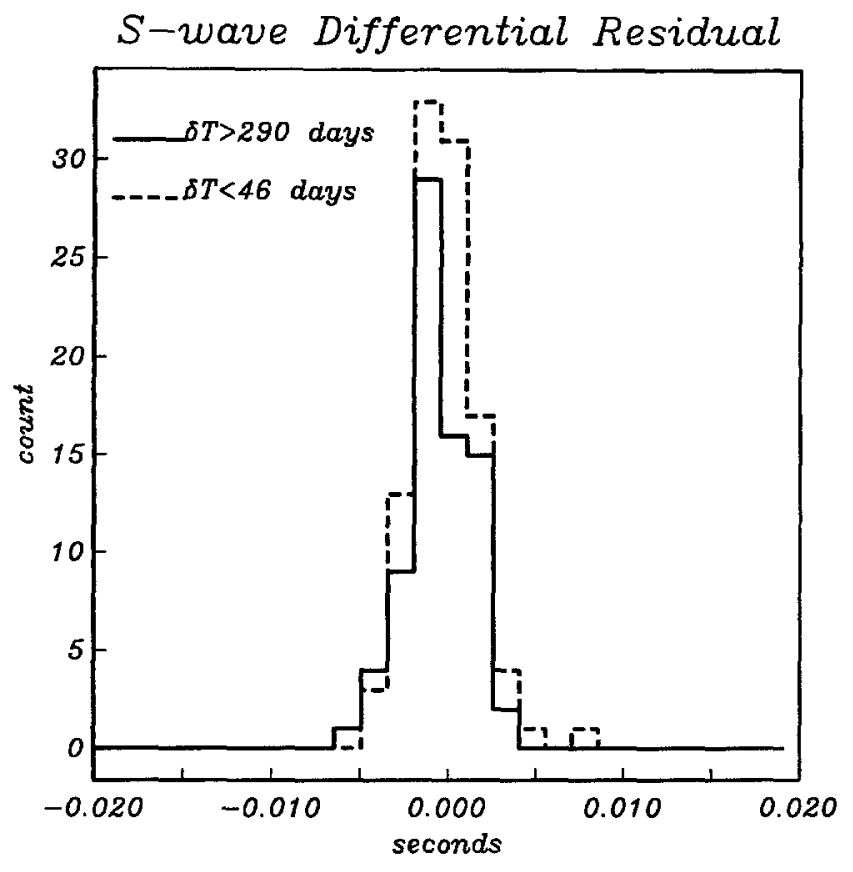

(b)

Figure 5. (b) Histograms of differential $S$-wave travel-time residuals for the subset of event pairs with $\delta T>290$ days (solid line) and the subset of event pairs with $\delta T<46$ days (dashed line). The standard deviations are $\sigma=0.002 \mathrm{sec}$ for both $S$ wave residual distributions. histograms of the differential $P$-wave travel-time residuals for the 12 pairs of long-time separation events to histograms for the short-time separation events. For both sets of events, the histograms show a similar spread with a standard deviation in the $P$ - and $S$-wave residuals of $0.002 \mathrm{sec}$ (half the sampling period). A widespread velocity change affecting the entire network would be difficult to resolve since the mean change in travel times would be removed in an adjustment to the relative origin times of the events. However, if there were significant changes in seismic velocity affecting a portion of the network over long time intervals, we would expect that there would be a much larger variation in travel-time residuals for the earthquake pairs with large time separations than for earthquake pairs with short time separations. The distribution of travel-time residuals for events with short time separation should serve as an indication of the noise level in the process, and is only slightly larger than the estimate of the picking error derived from the widths of the cross-correlation function peaks. Figure 5 b shows a nearly identical distribution for the $S$-wave differential travel-time residuals. The superiority of the cross-correlation method of picking relative arrival times compared to conventional arrival-time picking is demonstrated here, where the normally difficult-to-pick $S$-wave arrival times (e.g., see Gomberg et al., 1990) are as accurate as the $P$-wave arrival times. More importantly,

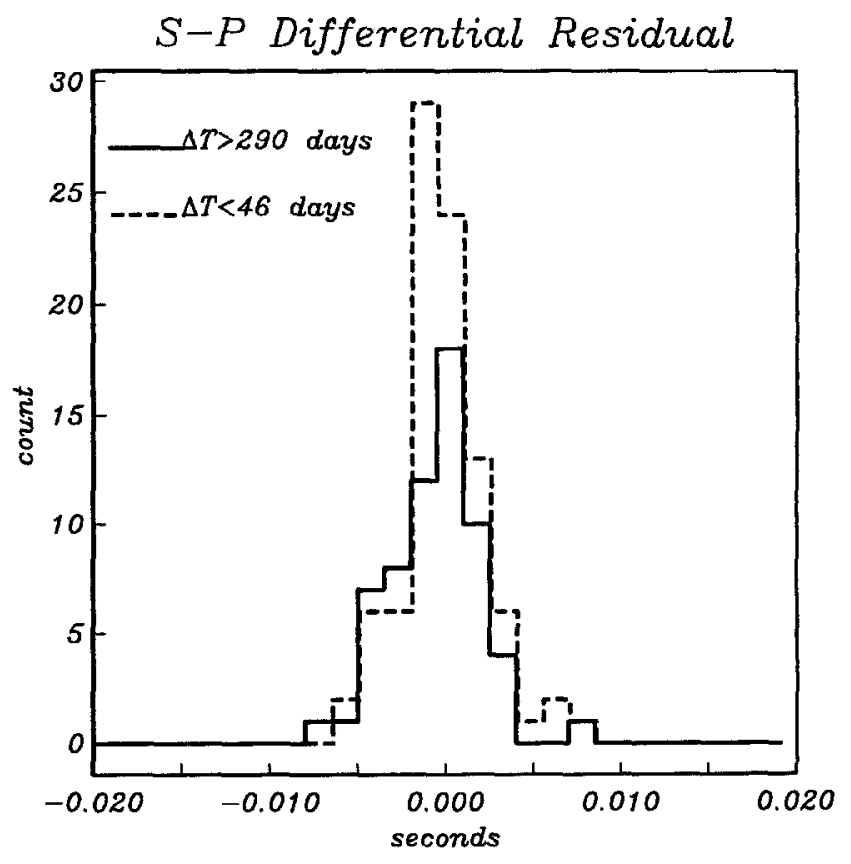

Figure 6. Histograms of differential $S-P$ time residuals for the subset of event pairs with $\delta T>$ 290 days (solid line, $\sigma=0.0026 \mathrm{sec}$ ) and the subset of event pairs with $\delta T<46$ days (dashed line, $\sigma=0.0024 \mathrm{sec}$ ). These residuals are corrected for offsets in event origin times and for differences in event locations. 
this indicates that there is no evidence for temporal changes in $P$ or $S$ velocities that are larger than the probable errors in the data.

The approximately half-sample accuracy of the method suggests that asynchronous sampling (see above) might be a significant source of error. We investigated this possibility by computing relative locations using $S-P$ times alone. If there was a sampling delay at one station, it would affect the $P$ - and $S$-wave arrival times at that station equally. Thus, if we calculate the relative $S-P$ times at a station for a pair of events, any sampling delay error will be removed. We then calculated the relative location of the pair of events using the relative $S-P$ times and removed the effect of the difference in location to derive a set of differential $S-P$ time residuals. Histograms for differential $S-P$ time residuals are displayed in Figure 6, and show a standard deviation of $0.003 \mathrm{sec}$ for event pairs with long time separations as well as for event pairs with short time separations. This demonstrates once again that there is no evidence for significant temporal changes in seismic velocity that are greater than the noise in the data. The standard deviation of $0.003 \mathrm{sec}$ is slightly larger than the standard deviation of $0.002 \mathrm{sec}$ for the individual $P$ and $S$ residuals, indicating that the $P$ and $S$

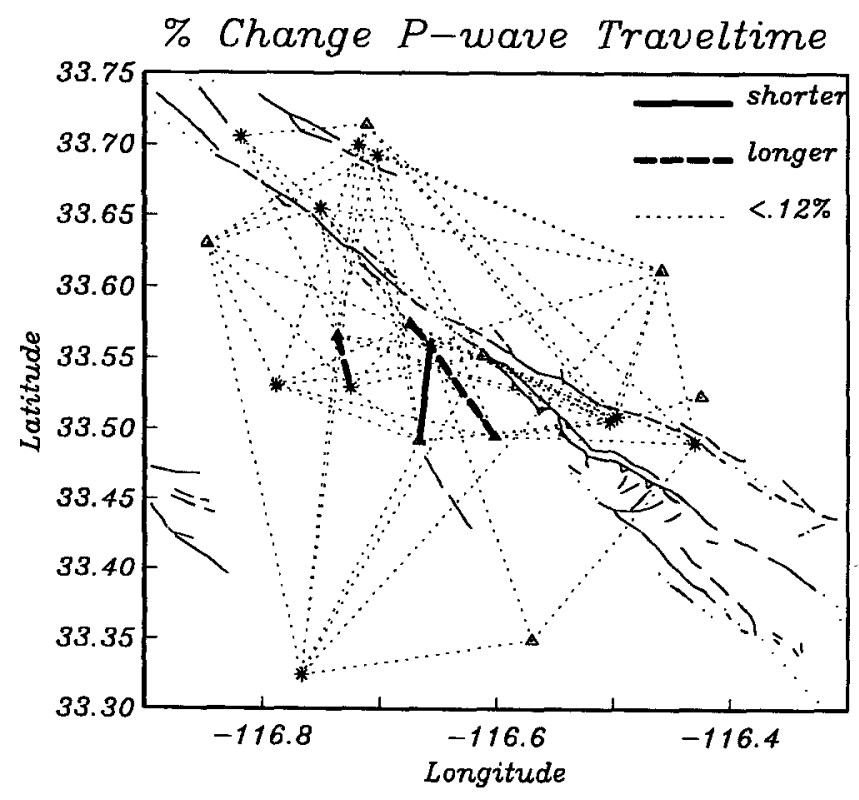

(a)

Figure 7. (a) Ray paths for large $\delta T$ event pairs with a percent change in $P$-wave travel time greater than two standard deviations of the small $\delta T$ residuals, $2 \sigma=0.12 \%$. Solid lines indicate ray paths with an apparent increase in seismic velocity (shorter times), dashed lines indicate ray paths with an apparent decrease in velocity (longer times), and dotted lines indicate ray paths with a change less than $2 \sigma$. residuals are largely uncorrelated and that an asynchronous sampling error is not the limiting error source in the measurements.

There is no clear evidence in the dataset as a whole for any temporal change in velocity, but the histograms plotted in Figure 6 could miss a localized anomaly that is sampled by only a few ray paths. To test for this possibility, we examined the residual patterns to see if we could identify any spatially coherent signals in the data. Figure 7 shows the ray paths sampled by the event pairs with large time separations. Ray paths that exhibit a percentage decrease in travel time greater than twice the standard deviation of the percent change of the residuals for event pairs with small time separation are shown as thick solid lines. Ray paths that exhibit a percentage increase in travel time are shown as thick dashed lines, while ray paths that exhibit a percentage change less than 2 standard deviations are shown as thin dotted lines. There are very few $P$ - or $S$-wave paths that show any significant change, and these are not obviously spatially correlated. Figure 8 shows a similar plot for the $S-P$ travel times. The largest change here is associated with an event that has few arrivals and thus is very poorly constrained.

Next we investigate any systematic time dependence

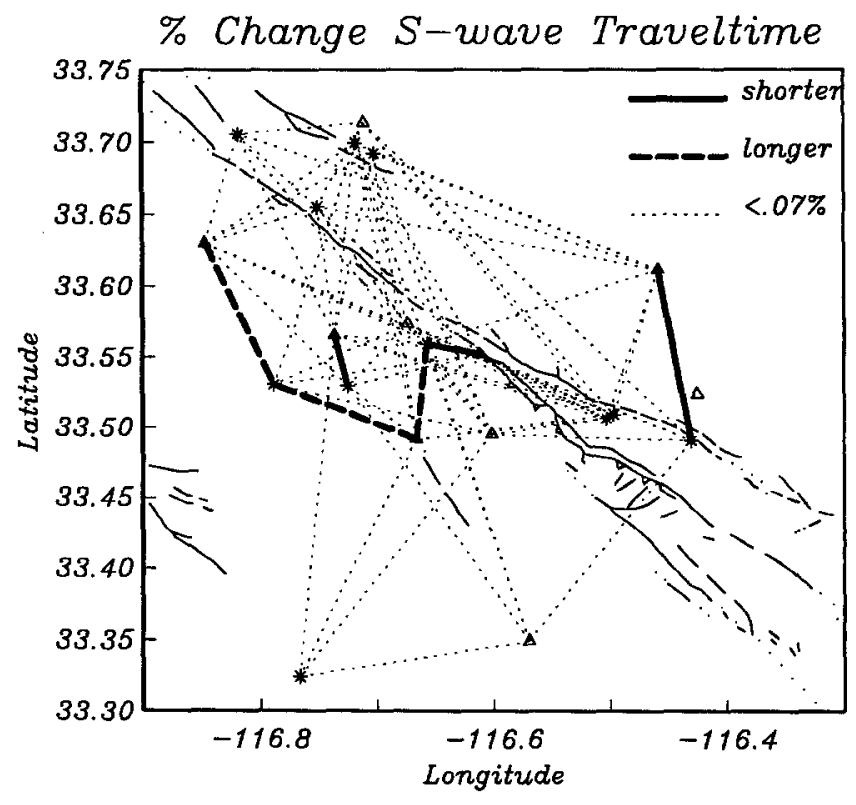

(b)

Figure 7. (b) Ray paths for large $\delta T$ event pairs with a percent change in $S$-wave travel time greater than two standard deviations of the small $\delta T$ residuals, $2 \sigma=0.07 \%$. Solid lines indicate ray paths with an apparent increase in velocity (shorter times), dashed lines indicate ray paths with an apparent decrease in velocity (longer times), and dotted lines indicate ray paths with a change less than $2 \sigma$. 
of the differential travel-time residuals and examine their possible relation to the times of large regional earthquakes. There have been eight events within $100 \mathrm{~km}$ of the Anza seismic gap with magnitude greater than 5 during the $9 \mathrm{yr}$ spanned by this study (Fig. 9). These include the $1986 M_{L}=5.6$ North Palm Springs, the $1986 M_{L}=$ 5.4 Oceanside, the $1987 M_{L}=5.9,5.3$ Whittier Narrows, the $1987 M_{L}=5.9,6.1$ Superstition Hills, the $1990 M_{L}=5.2$ Upland, and the $1991 M_{L}=5.8$ Sierra Madre earthquakes. Crampin et al. (1990) reported seeing a large change in shear-wave splitting delay times at station KNW of the Anza network at approximately the time of the North Palm Springs event. However, Aster et al. $(1990,1991)$ were unable to reproduce this result, and showed that similar earthquake pairs from the same dataset do not exhibit significant temporal changes in shearwave splitting. Similar earthquake pairs permit analysis that is free from the large uncertainties and potential biases associated with measuring individual delay times from dissimilar events. This study also finds no premonitory changes in seismic velocity associated with the North Palm Springs event.

In Figure 10a, we show for three stations a line for each event pair connecting the time of the two events, plotted versus the apparent percent change in $P$-wave travel time. The short vertical lines correspond to event pairs that are very closely spaced in time and thus illustrate the magnitude of the uncertainty in the measure-

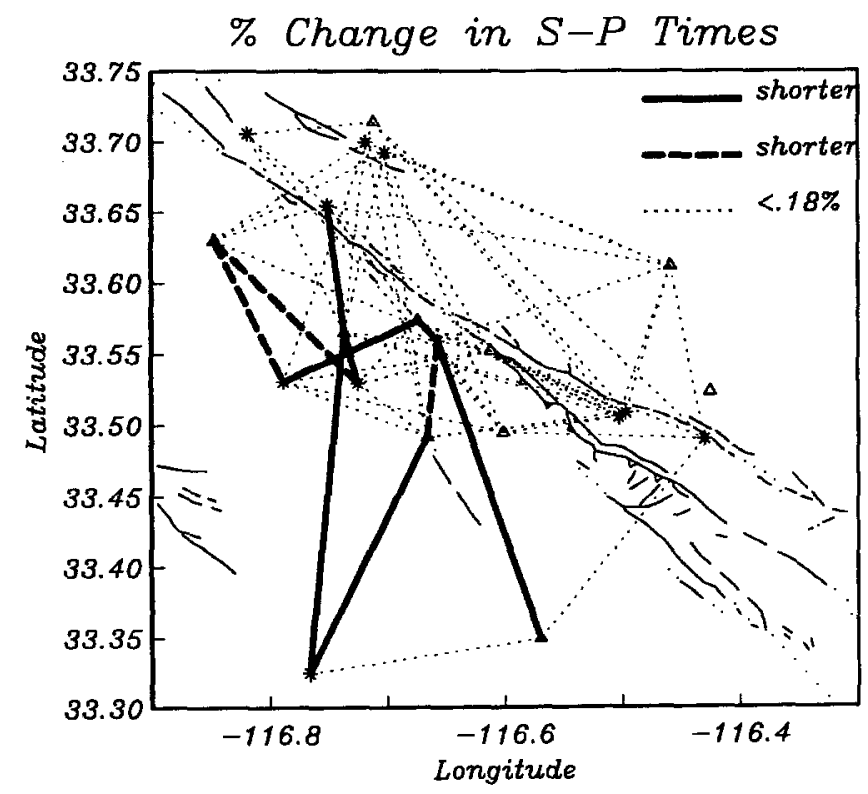

Figure 8. Ray paths for large $\delta T$ event pairs with a percent change in $S-P$ time greater than two standard deviations of the small $\delta T$ residuals, $2 \sigma$ $=0.07 \%$. Solid lines indicate ray paths with an apparent decrease in $S-P$ time and dashed lines indicate ray paths with an apparent increase in $S-P$ time, and dotted lines indicate ray paths with a change less than $2 \sigma$. ments. The dashed vertical lines correspond to the times of regional events with magnitudes greater than 5.0 (Fig. 9). There are positive changes as well as negative changes ascribed to these three stations and there is no obvious association with the time of major events. This is also true for the percent change in $S$-wave residuals shown in Figure 10b, as well as for the other seven stations not shown.

In summary, a thorough investigation of a variety of source-receiver paths that sample the crust near the Anza seismic gap finds no evidence for an accumulated temporal change in seismic velocity over long time scales. The examination of similar earthquake pairs separated by very short times provides a reliable estimate of the noise level of the data.

\section{Resolution}

If there is any localized change in seismic travel times, it cannot be resolved by these data. The question remains as to how small a signal is detectable given our dataset. There are two major factors inhibiting our ability to resolve a velocity change. One is the background level of noise, which we estimated from our investigation of similar event pairs with small time separations to be 0.002 $\mathrm{sec}$ in travel time. The second factor is the trade-off between event mislocation and changes in seismic velocity.

We note that it would be very difficult to detect a change in velocity if it occurred uniformly throughout

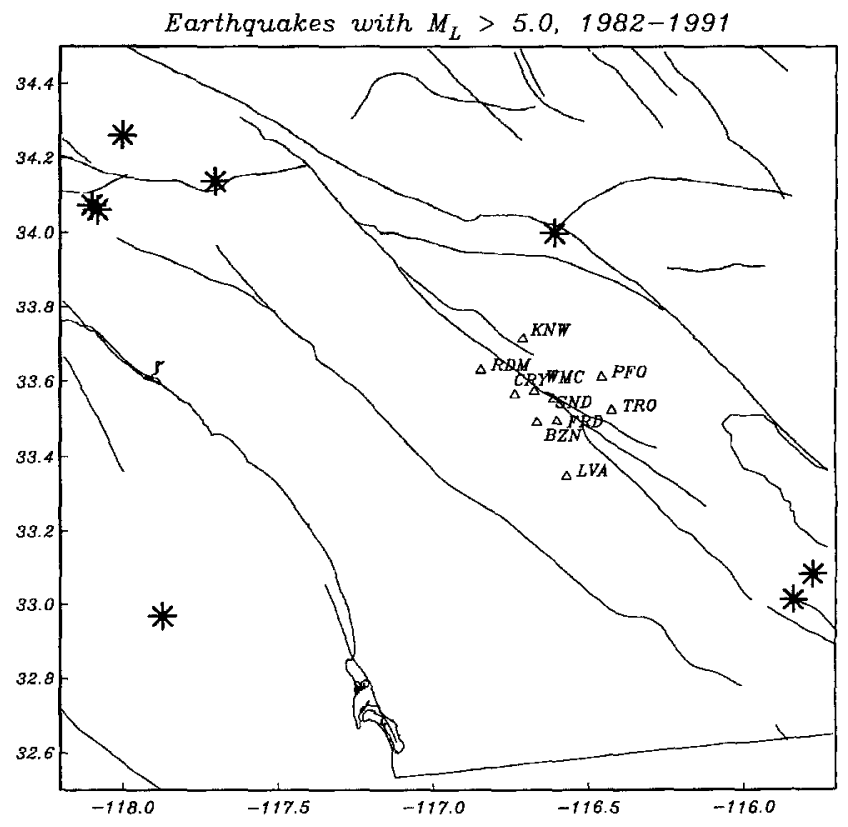

Figure 9. Earthquakes in southern California with $M_{L}>5$ that occurred during approximately the first 9 yr of operation of the Anza Seismic Network (the time spanned by this study). 

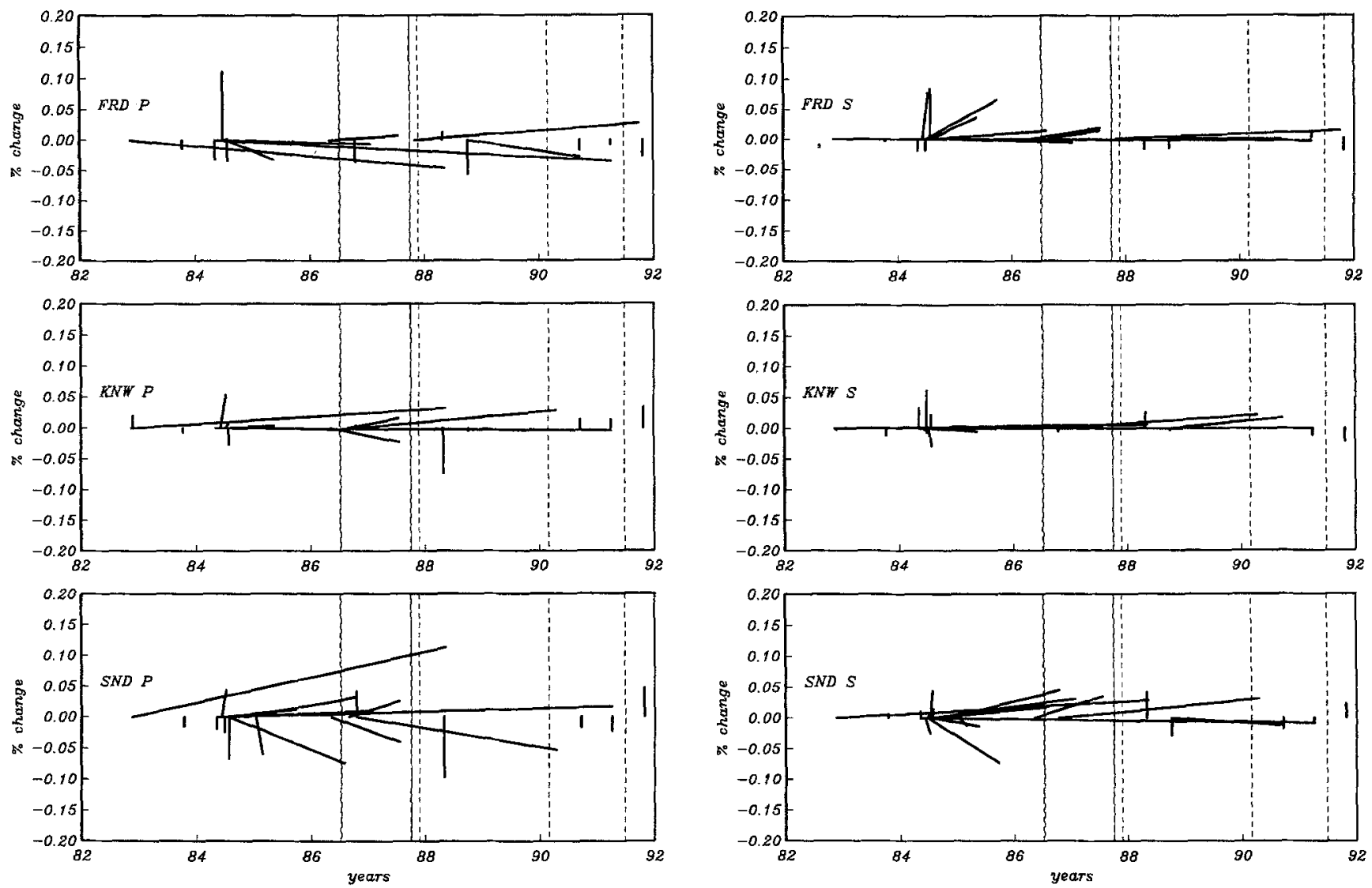

(a)

(b)

Figure 10. (a) Time history of apparent $P$-wave travel-time change at stations FRD, KNW, and SND. A solid line connects the two events of each pair indicating the apparent change in travel time between the events. The short vertical lines correspond to event pairs that are very closely spaced in time and illustrate the magnitude of the uncertainty in the measurements. The dashed vertical lines correspond to the times of the regional events with $M_{L}>=5.0$ (Fig. 9). (b) Time history of apparent $S$-wave travel-time change at stations FRD, KNW, and SND. A solid line connects the two events of each pair indicating the percent change in travel time between the events.

the sampled region, especially since there are only a small number of stations in the array. Such a change could be mistaken for an approximate difference in depth between the two events. Nothing can be said about the probability of such a change from this method. We thus wish to determine the ability of the method to resolve a localized change in velocity, for example, a change which only affects one station, perhaps nearest the fault. We test this scenario by creating a synthetic set of arrival times for two colocated events, where the travel time to one of the stations has been perturbed by a given amount. To these synthetic arrival times, we add Gaussian noise with standard deviation $0.002 \mathrm{sec}$, which is approximately the random noise level in the data. The relative location is calculated and the remaining residuals are examined for traces of the input travel-time perturbation. Because there is noise in the arrival times and because of the nonuniqueness of the problem, some of the travel-time re- sidual can be accommodated by giving the two events a small source separation, and it will thus only be possible to recover a portion of the anomalous travel-time residual at the station. Figure 11a shows an experiment where we calculate the bias in event separation that results when a given percent change in velocity is assigned to one of the source-station ray paths. For this example, we used the station source geometry that was available for the event pair with a 2512-day separation (event pair number 26, Figs. 1 and 2). In general, the calculated event separation distance increases as the size of the velocity change increases, though with considerable scatter. This limits our ability to recover the velocity change from the differential travel time at a station. Figure $11 \mathrm{~b}$ shows the size of the percent travel-time change recovered for a given input change in percent velocity. The dashed line shows the differential travel-time residual for the station whose travel time was perturbed (WMC) and the solid 


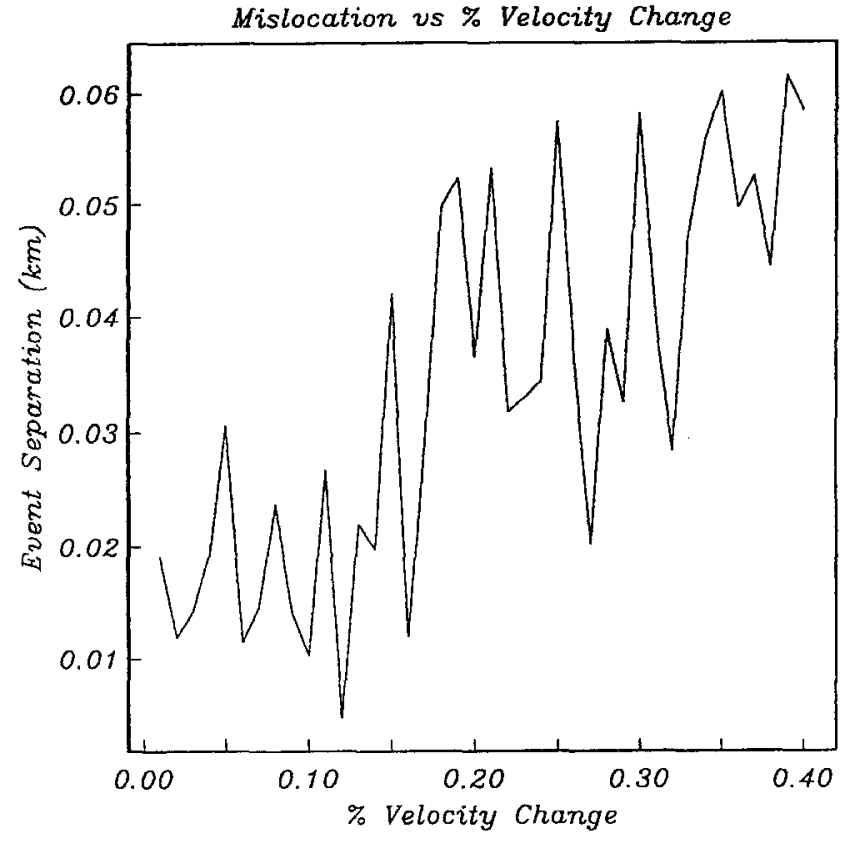

(a)

Figure 11. (a) Results of a synthetic test illustrating the trade-off between a localized change in seismic velocity and the resulting difference in event location produced by the altered differential travel times. Gaussian noise with $\sigma=0.002 \mathrm{sec}$ has been added to the synthetic travel times. Although there is some scatter, it is clear that some of the signal from a change in velocity can be accommodated by a separation of the hypocenters, limiting the resolution of velocity changes.

lines show the differential travel-time residual for the other stations of the network. It can be seen that once the percent velocity change is greater than about $0.2 \%$, the dashed line can be distinguished from the errors in traveltime residuals at the other stations. The ability of the method to detect a travel-time change is very dependent on the source-receiver geometry and the lateral extent of the velocity anomaly, but this example serves to demonstrate approximately what size localized velocity change can be "hidden" by the timing uncertainties in the data and by the location/velocity change trade-off. We can be reasonably confident that this network configuration can detect localized velocity changes greater than $0.2 \%$ and can state that if there are any localized temporal changes in velocity in the Anza region along these modeled ray paths, they must be smaller than $0.2 \%$.

\section{Discussion}

Two recent studies of aftershock zones using similar earthquakes on the Calaveras fault (Poupinet et al., 1984; Ellsworth et al., 1987) and the Loma Prieta fault zone (Ellsworth et al., 1992) have revealed significant changes

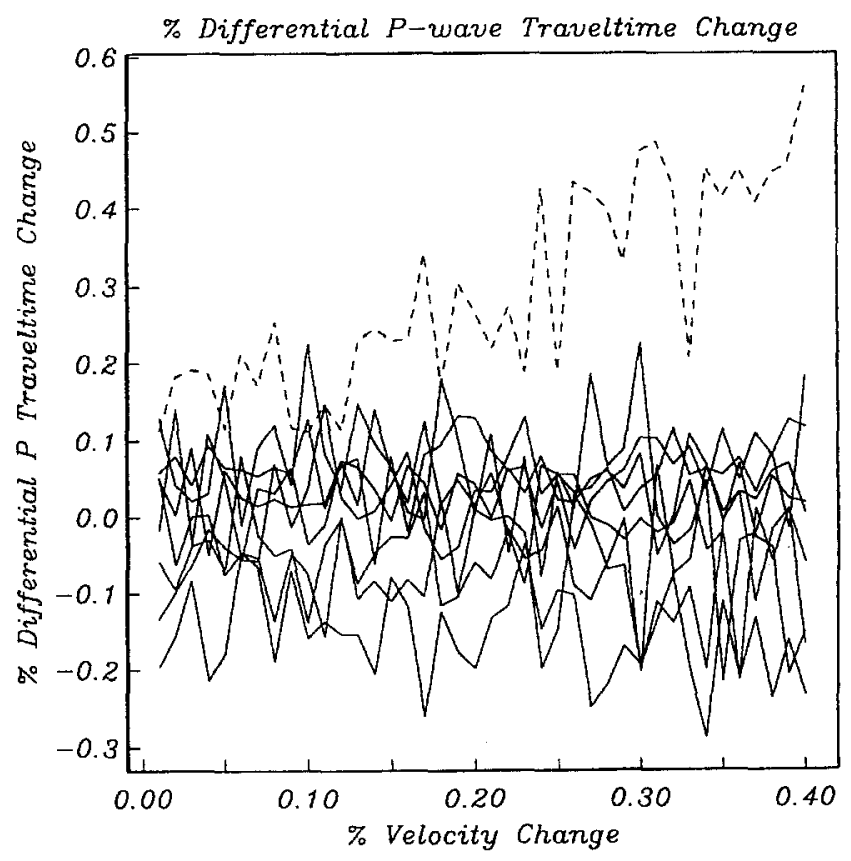

(b)

Figure 11. (b) Percent travel-time change recovered for a given input change in velocity for paths to one station (dashed line). Solid lines show the errors in the percent change in travel time calculated at the remaining stations caused by the mislocation error. A velocity change greater than about $0.2 \%$ can be detected above this noise level.

in travel times for some ray paths crossing the rupture zone of large earthquakes. These studies used a frequency-domain, moving-window cross-correlation scheme that allowed them to calculate time delays for arrivals later in the coda than the direct $S$ wave. We did not find any systematic change in direct $P$ - or $S$-wave arrival times at Anza. This may be due to the fact that we are not measuring travel times in a region close to a large event like Coyote Lake or Loma Prieta - the Anza Seismic Gap has not ruptured in a major earthquake since the deployment of the Anza Network. Likewise, the Parkfield segment of the San Andreas did not show any detectable change (Nadeau et al., 1992). As far as an indicator of premonitory changes in velocity, we have shown that there have been no detectable changes at Anza at the $0.2 \%$ level occurring in the last $9 \mathrm{yr}$. This study does not address the possibility of travel-time changes in the $P$ - or $S$-wave coda; we are currently examining the coda for possible temporal variations in coda $Q$ at Anza (Aster et al., 1992).

Similar earthquakes are very useful as repeatable seismic sources for investigations of possible temporal changes in seismic properties. The presence of events within the seismogenic region provides a better sampling of the fault zone at depth than is normally possible from 
near-surface sources such as quarry blasts and vibroseis. Similar earthquakes are an especially effective tool for regions such as Anza where the seismicity is typically very clustered and provides a well-sampled time history. This study, together with the shear-wave splitting study of Aster et al. (1990, 1991), which also used similar event pairs, indicates that there have been no detectable changes in $P$ and $S$ velocity or seismic anisotropy at Anza. The limits on temporal variations from these studies are about an order of magnitude more accurate than would be possible using nonrepeatable sources.

\section{Conclusions}

A study of similar event pairs recorded during an approximately 9-yr period by the Anza Seismic Network shows that travel-time perturbations for event pairs separated by more than 290 days are not significantly larger than measurements made for pairs separated by less than 46 days. Results from the short-time-separation pairs indicate that the relative arrival time accuracy is $0.002 \mathrm{sec}$ for $P$ and $S$ waves. Synthetic experiments with similar station distributions show that a localized velocity change as small as $0.2 \%$ in the fault zone should be detectable with this dataset, whereas all of the measurements of travel-time perturbations are less than $0.15 \%$. Detailed studies of the spatial and temporal distribution of the ray paths do not identify any subsets of the data with reliable variations larger than this. Therefore, it is unlikely that there is a systematic temporal change in seismic velocity greater than $0.2 \%$ due to a localized accumulation of stress in this region in this 9-yr time period.

\section{Acknowledgments}

Funding for this study was provided by USGS Grant Numbers 1408-0001-G1767, 1434-93-G-2295, and 1434-92-G-2202. The authors would like to thank Frank Vernon for his interest and support in this project and his help in working with the Anza Network dataset.

\section{References}

Aggarwal, Y. P. (1973). Premonitory changes in seismic velocities and prediction of earthquakes, Nature 241, 101-104.

Aggarwal, Y. P., L. R. Sykes, D. W. Simpson, and P. G. Richards (1975). Spatial and temporal variations in $t s / t p$ and in $P$ wave residuals at Blue Mountain Lake, New York: application to earthquake prediction, J. Geophys. Res. 80, 718-732.

Anderson, D. and J. Whitcomb (1975). Time-dependent seismology, J. Geophys. Res. 80, 1497-1503.

Aster, R. C., J. Henton, J. Scott, P. Shearer, and F. Vernon (1992). Comparative coda analysis of similar earthquakes with large temporal separations near the Anza Seismic Gap, Southern California, $\operatorname{EOS~73,~} 361$.

Aster, R. C. and J. S. Scott (1993). Comprehensive characterization of waveform similarity in large microearthquake data sets, Bull. Seism. Soc. Am. 83, 1307-1314.

Aster, R. C., P. M. Shearer, and J. Berger (1990). Quantitative measurements of shear wave polarizations at the Anza Seismic Net- work, southern California: implications for shear wave splitting and earthquake prediction, J. Geophys. Res. 95, 12449-12473.

Aster, R. C., P. M. Shearer, and J. Berger (1991). Reply to Crampin et al., J. Geophys. Res. 96, 6415-6419.

Bak, P. and C. Tang (1989). Earthquakes as a self-organized critical phenomenon, J. Geophys. Res. 94, 15635-15637.

Berger, J., L. M. Baker, J. N. Brune, J. B. Fletcher, T. C. Hanks, and F. L. Vernon III (1984). The Anza array: a high-dynamic range, broadband, digitally radio telemetered seismic array, Bull. Seism. Soc. Am. 74, 1469-1481.

Bolt, B. (1977). Constancy of $\mathbf{P}$ travel times from Nevada explosions to Oroville Dam station 1970-1976, Bull. Seism. Soc. Am. 67, 27-32.

Boore, D. M., A. G. Lindh, T. V. McEvilly, and W. W. Tolmachoff (1975). A search for travel time changes associated with the Parkfield earthquake of 1966, Bull. Seism. Soc. Am. 65, 14071418.

Brune, J. N. (1979). Implications of earthquake triggering and rupture propagation for earthquake prediction based on premonitory phenomena, J. Geophys. Res. 84, 2195-2198.

Chou, C. W. and R. S. Crosson (1978). Search for time-dependent seismic $\mathbf{P}$ travel times from mining explosions near Centralia, Washington, Geophys. Res. Lett. 5, 97-100.

Crampin, S., D. C. Booth, R. Evans, S. Peacock, and J. Fletcher (1990). Changes in shear wave splitting at Anza near the time of the North Palm Springs earthquake, J. Geophys. Res. 95, 11197-11212.

Ellsworth, W. L., L. D. Dietz, J. Frechet, and G. Poupinet (1987). Preliminary results on the temporal stability of coda waves in central California from high-precision measurements of characteristic earthquakes, U.S. Geol. Surv. Open-File Rept. 87-591, 440-460.

Ellsworth, W. L., A. T. Cole, G. C. Beroza, and M. C. Verwoerd (1992). Changes in crustal wave velocity associated with the 1989 Loma Prieta, California earthquake, EOS 73, 360.

Fremont, M.-J. and S. D. Malone (1987). High precision relative locations of earthquakes at Mount St. Helens, Washington, $J$. Geophys. Res. 92, 10223-10236.

Gomberg, J. S., K. M. Shedlock, and S. W. Roecker (1990). The effect of S-wave arrival times on the accuracy of hypocenter estimation, Bull. Seism. Soc. Am. 80, 1605-1628.

Ito, Akihiko (1985). High resolution relative hypocenters of similar earthquakes by cross-spectral analysis method, J. Phys. Earth 33, 279-294.

Jordan, T. H. and K. A. Sverdrup (1981). Teleseismic location techniques and their application to earthquake clusters in the southcentral Pacific, Bull. Seism. Soc. Am. 71, 1105-1130.

Kagan, Y. Y. and L. Knopoff (1981). Stochastic synthesis of earthquake catalogs, J. Geophys. Res. 86, 2853-2862.

Kagan, Y. Y. and L. Knopoff (1987). Random stress and earthquake statistics: time dependence, Geophys. J. Int. 88, 723-731.

Kanamori, H. and G. Fuis (1976). Variation of $P$ wave velocity before and after the Galway Lake earthquake $\left(M_{L}=5.2\right)$ and the Goat Mountain earthquakes $\left(M_{L}=4.7,4.7\right), 1975$, in the Mojave desert, California, Bull. Seism. Soc. Am. 66, 2027-2037.

Leary, P. C., P. E. Malin, R. A. Phinney, T. Brocher, and R. VonColln (1979). Systematic monitoring of millisecond travel time variations near Palmdale, California, J. Geophys. Res. 84, 659-666.

Leary, P. C. and P. E. Malin (1982). Millisecond accurate monitoring of seismic travel times over 13- and 18-kilometer baselines, $J$. Geophys. Res. 87, 6919-6930.

Lindh, A. G., D. A. Lockner, and W. H. K. Lee (1978). Velocity anomalies: an alternative explanation, Bull. Seism. Soc. Am. 68, 721-734.

McEvilly, T. V. and L. R. Johnson (1974). Stability of $P$ and $S$ velocities from central California quarry blasts, Bull. Seism. Soc. Am. 64, 343-353. 
Nadeau, R. M., and M. Antolik, and T. V. McEvilly (1992). A search for temporal changes in travel times and polarization at Parkfield using earthquake sources, Seism. Res. Lett. 63, 62.

Nur, A. (1972). Dilatancy, pore fluids and premonitory variations of ts/tp travel times, Bull. Seism. Soc. Am. 62, 1217-1222.

Poupinet, G., W. L. Ellsworth, and J. Frechet (1984). Monitoring velocity variations in the crust using earthquake doublets: an application to the Calaveras fault, California, J. Geophys. Res. 89, 5719-5731.

Robinson, R., R. L. Wesson, and W. L. Ellsworth (1974). Variations of $\mathbf{P}$ wave velocity before the Bear Valley, California, earthquake of 24 February 1972, Science 184, 1281-1283.

Rundle, J. B., A physical model for earthquakes: I. fluctuations and interactions, J. Geophys. Res. 93, 6237-6254.

Scholz, C., L. Sykes, and Y. Aggarwal (1973). Earthquake prediction: a physical basis, Science 181, 803-810.

Semenov, A. M. (1969). Variations in the travel time of transverse and longitudinal waves before violent earthquakes, Izv. Acad. Sci. USSR Phys. Solid Earth 4, 245-248.

Vernon III, F. L. (1989). Analysis of data recorded on the Anza Seismic Network, Ph.D. Thesis, University of California, San Diego.

Whitcomb, J. H., J. E. Garmany, and D. L. Anderson (1973). Earth- quake prediction: variation of seismic velocities before the San Francisco (sic) earthquake, Science 180, 632-635.

Working Group on California Earthquake Probabilities (1988). Probabilities of large earthquakes occurring in California on the San Andreas fault, U.S. Geol. Surv. Open-File Rept. 88-398, 1-62.

Seismological Laboratory

California Institute of Technology

Pasadena, California 91125

(J.S.H.)

Institute of Geophysics and Planetary Physics

Scripps Institution of Oceanography

La Jolla, California 92093

(P.M.S.)

Department of Geoscience and Geophysical Research Center New Mexico Institute of Mining and Technology Socorro, New Mexico 87801

(R.C.A.)

Manuscript received 6 July 1993. 